\title{
Испытания на восприимчивость к электромагнитному полю - новые решения
}

\author{
Д. Кондрашов ${ }^{1}$, А. Шостак ${ }^{2}$, П. Дейкстра ${ }^{3}$
}

УДК 621.317| ВАК 05.11.01

\begin{abstract}
К оборудованию, используемому для испытаний на электромагнитную совместимость (ЭМС), предъявляется целый ряд требований: по уровню напряженности генерируемых электромагнитных полей, точности измерений, времени проведения испытаний, автоматизации и др. Недавно был разработан ряд эффективных решений - генераторов поля, датчиков поля и систем для испытаний на ЭМС, - позволяющих проводить проверку восприимчивости радиоэлектронного оборудования к мощным электромагнитным полям в соответствии с современными требованиями.
\end{abstract}

лассическая система, на основе которой проводят испытания в соответствии с ГОСТ 30804.4.3-2013, обладает рядом недостатков [1]:

- высоким КСВН, обусловленным компактностью ЭМС-антенн, из-за которой возникает компромисс между согласованием, эффективностью (КПД) и размерами антенн;

- ограничениями, присущими логопериодическим и рупорным антеннам, используемым при проведении испытаний. Логопериодическая антенна имеет крупные габариты, а также не обладает должным уровнем усиления, что требует больше мощности для создания однородного поля и достижения необходимых по стандарту значений напряженности поля в измерительных точках, прописанных в стандарте. Рупорная антенна не позволяет на трехметровом расстоянии облучать область 1,5×1,5 м для формирования однородного поля \pm 6 дБ из-за узкого луча диаграммы направленности (рис. 1);

- потерями в кабелях, каскадах усилителя, направленном ответвителе. Это в итоге приводит к существенным потерям, вследствие чего необходимо применять более мощный и дорогостоящий усилитель;

- для минимизации потерь необходимо размещать стойки с усилителями непосредственно в безэховой камере (БэК), что, во-первых, накладывает

ООО “Остек-Электро», начальник группы волновых процессов, Kondrashov.D@ostec-group.ru.

ООО "Остек-Электро", специалист технического сопровождения, Shostak.A@ostec-group.ru.

DARE!! Instruments (Нидерланды), технический директор, pady@dare.nl. требования по устойчивости к воздействиям электромагнитного поля на стойку и, во-вторых, значительно влияет на однородность создаваемого поля, особенно при небольших размерах БЭК.

Учитывая эти недостатки, компания DARE!! Instruments разработала систему (генератор поля), которая интегрирована в единый корпус (рис. 2) и включает активную антенную решетку (AАP), усилители и измерители мощности. При использовании данной системы выполняется суммирование полей, создаваемых каждым элементом AАР, и контроль излучаемой и отраженной мощности

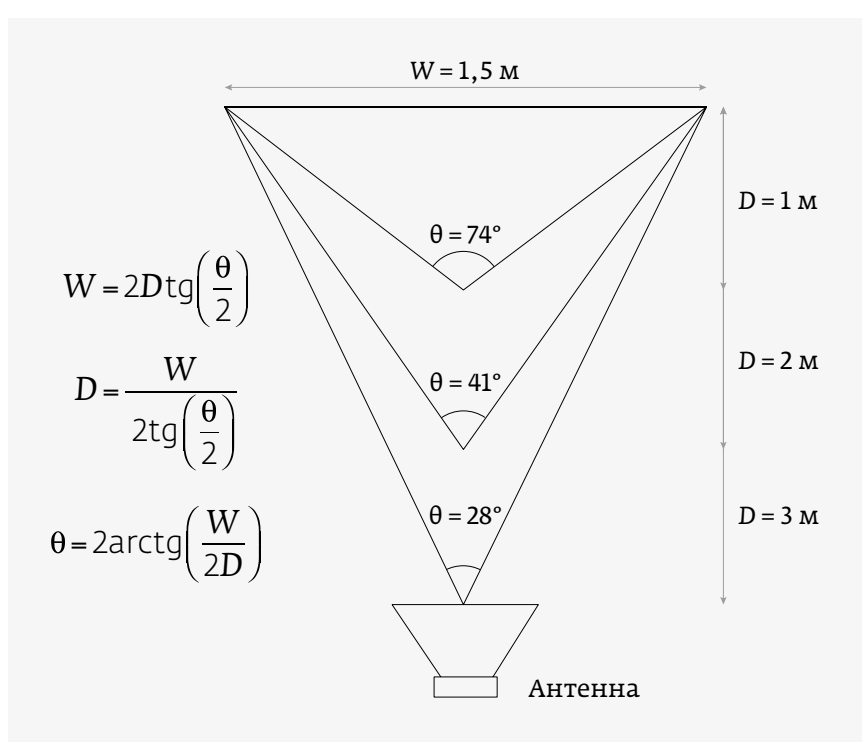

Рис. 1. Зависимость ширины ДН рупорной антенны от измерительного расстояния. $W$ - ширина плоскости однородного поля, D - измерительное расстояние, $\theta$ - угол раскрыва рупорной антенны 


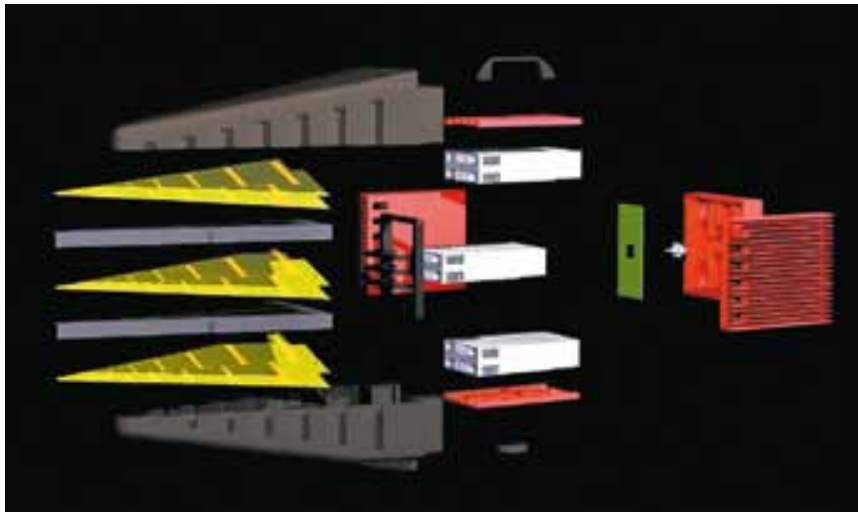

Рис. 2. Генератор поля RFS2006BR

встроенных усилителей. С помощью этой технологии первоначально ставилась цель получить гарантированное значение напряженности поля на расстояниях один и три метра с учетом амплитудной модуляции в соответствии со стандартом ГОСТ 30804.4.3-2013. Максимальный уровень напряженности поля, который был доступен на начало 2017 года - 54 В / м в диапазоне частот 800 МГц 6 ГГц на расстоянии 1 м с перепадом 0-6 дБ, что полностью удовлетворяло требованиям ГОСТ 30804.4.3-2013. Однако впоследствии инженерам удалось добиться значения напряженности поля в 60 В / м в аналогичном частотном диапазоне. Результаты испытаний с применением ААР при вертикальной и горизонтальной поляризации представлены на рис. 3. Можно отметить стабильность уровня создаваемой напряженности поля при обоих видах поляризации. Уровень сигнала, поступающего с генератора СВЧ-сигналов на вход генератора поля, показывает, что существует запас для усиления. Это позволит в будущем повысить значения гарантированно создаваемой напряженности.

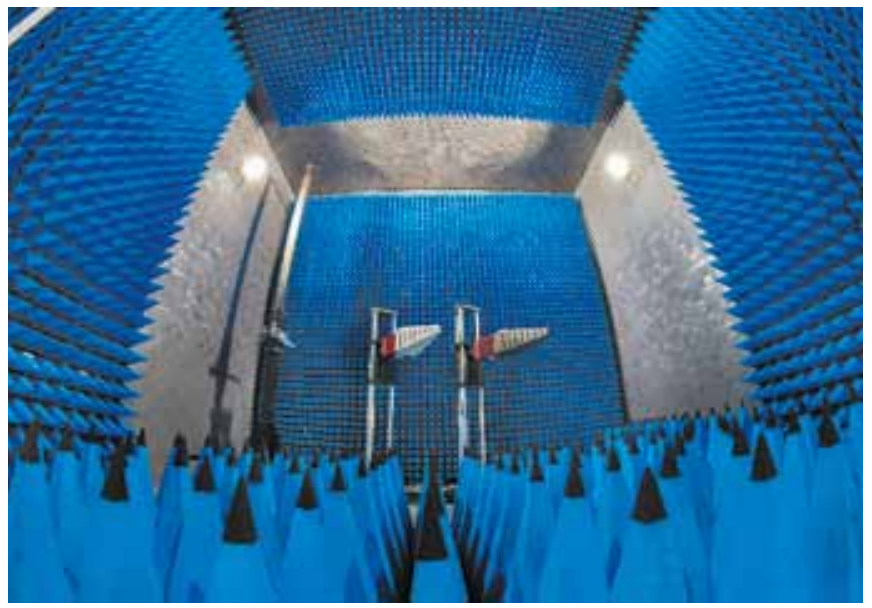

Рис. 4. Генераторы поля RFS2006BR и RFS2018BR

Максимально возможный уровень напряженности, которого удалось достичь с использованием данной ААР в диапазоне от 800 МГц до б ГГц - это 90 В / м в точке на расстоянии 1 м, что позволяет проводить испытания по авиационным и автомобильным стандартам.

Применение концепции ААР для испытаний на устойчивость к радиочастотному электромагнитному полю хорошо зарекомендовало себя и используется уже большим количеством современных лабораторий во многих странах: от США до Южной Кореи.

В конце 2017 года ООО "Остек-Электро" анонсировало новый комплект для испытаний на восприимчивость к РЧ-полю в диапазоне частот от 800 МГц до 18 ГГц, который построен на базе двух генераторов поля: RFS2006BR (800 МГц - 6ГГц) и RFS2018BR (6ГГц - 18 ГГц) (рис. 4). Принцип суммирования полей, создаваемых элементами ААР, остался прежним, однако данная система позволила создавать поля с гарантированным значением

- Мощность, подаваемая на антенну - Мощность сигнала, поступающего с генератора СвЧ-сигналов - Напряженность
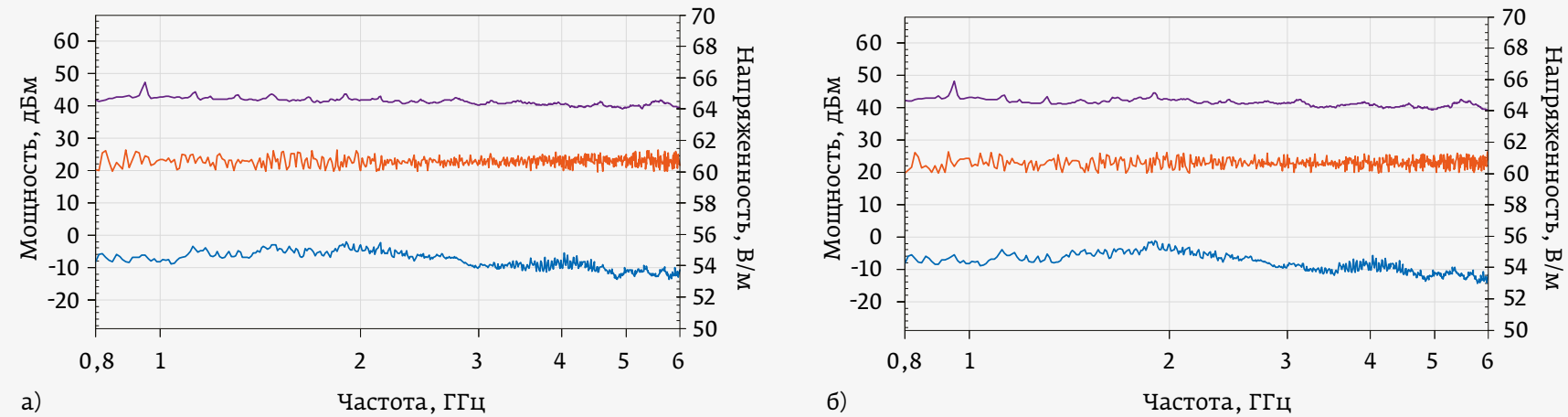

Рис. 3. Напряженность, создаваемая генератором поля RFS2006BR при горизонтальной (а) и вертикальной (б) поляризации 


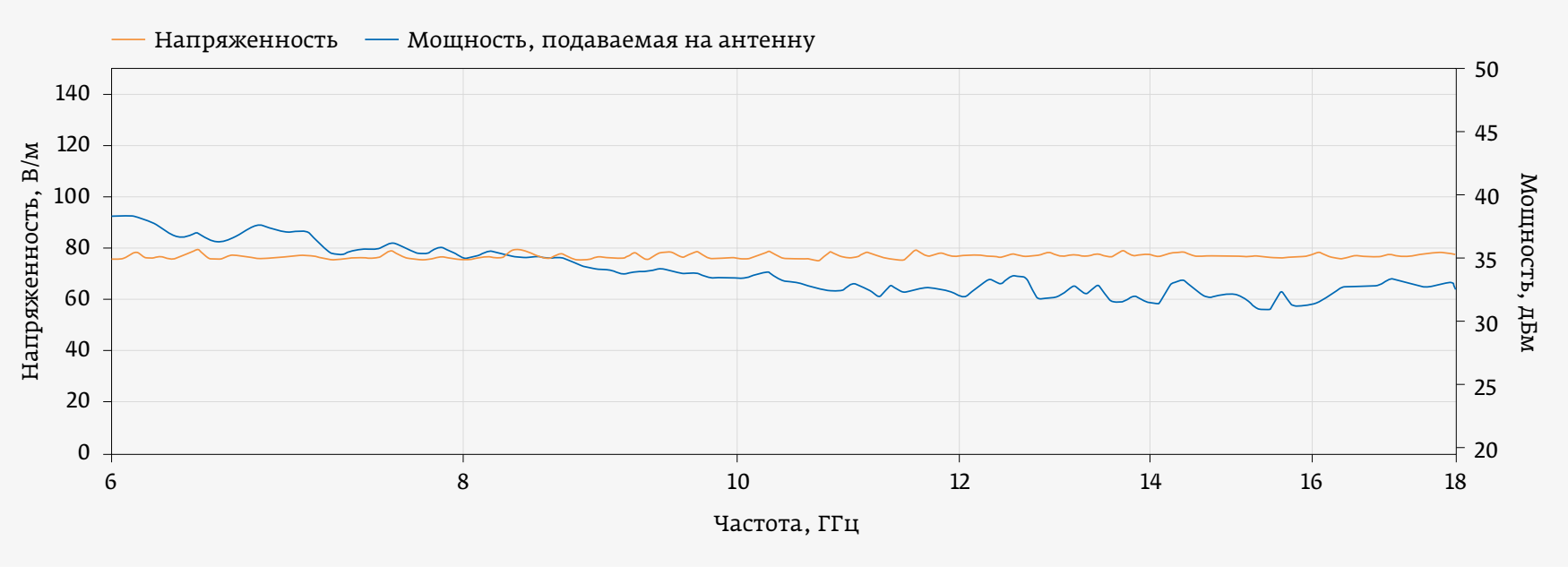

Рис. 5. Напряженность, создаваемая генератором поля RFS2018BR на измерительном расстоянии 1 м при горизонтальной поляризации

напряженности 75 В/м (рис. 5) и максимальным значением - свыше 150 В / м (рис. 6) в точке на измерительном расстоянии $1 \mathrm{~m}$.

После доработки генератора поля RFS2018BR в 2018 году появилась возможность гарантированно создавать напряженность поля до 100 В / м во всем диапазоне рабочих частот генератора, что позволяет использовать его для проведения испытаний по ряду автомобильных стандарTOB, к примеру, ISO 11451-2. Данная модернизация была осуществлена за счет уменьшения длины элементов АAP, а также использования нового материала для радиопрозрачного колпака генератора RFS2018BR.

Таким образом, используя данный комплект из двух генераторов поля, совместно перекрывающих диапазон частот от 800 МГц до 18 ГГц, можно удовлетворить

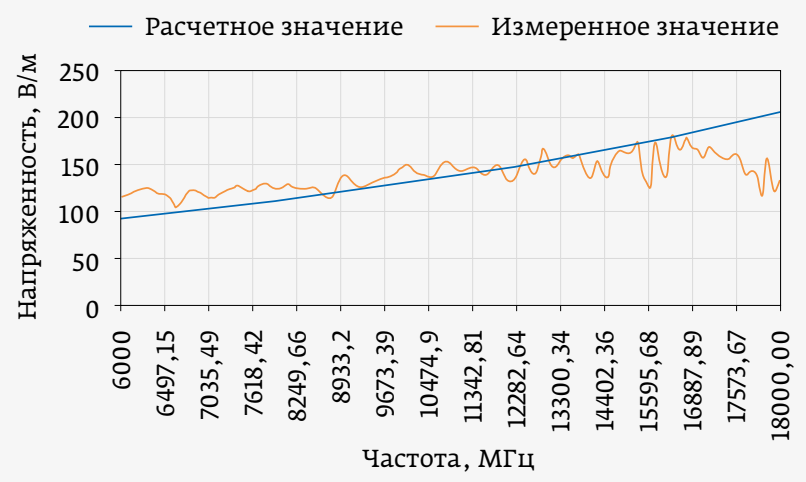

Рис. 6. Напряженность, создаваемая генератором поля RFS2018BR на расстоянии 1 м в частотном диапазоне 6-18 ГГц требования отечественных и зарубежных коммерческих, автомобильных и специализированных стандартов. Это позволяет существенно экономить при приобретении оборудования, а также предлагает более широкие возможности для проведения предварительных и сертификационных испытаний.

Учитывая потенциальные возможности суммирования полей, создаваемых элементами АAР, есть следующие перспективы их использования:

- доработка генератора для достижения стабильного уровня напряженности поля в 150 В/ м в безэховой камере. Это позволяет проводить испытания по авиационному стандарту KT-160D (раздел 20), требующему высоких уровней воздействия;

- использование генератора в реверберационных камерах для достижения уровня напряженности поля выше 200 В / м. Это дает возможность проводить испытания по специализированным стандартам, например по MIL-STD-461G (раздел RS103);

- увеличение верхней границы частотного диапазона генераторов поля до 67 ГГц для испытаний в аэрокосмической промышленности.

Однако, несмотря на используемый подход к облучению тестируемого устройства, наиболее важными задачами остаются калибровка испытательного стенда и точные измерения характеристик однородности поля используемой безэховой камеры, а также оптимальный выбор типа применяемого датчика напряженности поля.

На рис. 7 изображены кривые зависимости мощности, подаваемой на антенну, от частоты для трех датчиков поля [2]. Одна кривая для каждого датчика соответствует напряженности поля 100 В/м, другая - напряженности 200 В / м. Как видно из рис. 7, на частотах от 3 ГГц наблюдается значительное изменение мощности с частотой, 


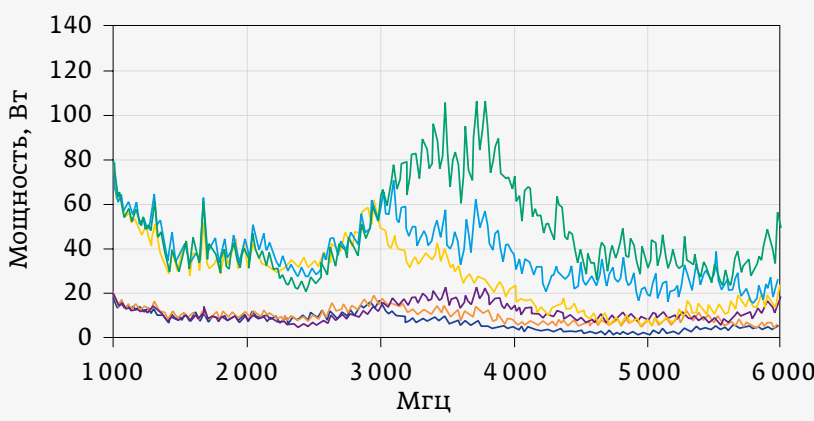

\begin{tabular}{rr}
$-100 \mathrm{~B} /$ м Датчик №1 & $200 \mathrm{~B} / \mathrm{M}$ Датчик №1 \\
\hline $100 \mathrm{~B} / \mathrm{M}$ Датчик №2 & $200 \mathrm{~B} / \mathrm{M}$ Датчик №2 \\
$-100 \mathrm{~B} / \mathrm{M}$ Датчик №3 & $200 \mathrm{~B} / \mathrm{M}$ Датчик №3
\end{tabular}

Рис. 7. Зависимость мощности, подаваемой на антенну, от частоты при горизонтальной поляризации для различных датчиков

несмотря на то, что датчики отображают одинаковое значение напряженности поля.

На рис. 8 приведены зависимости погрешности измерений напряженности от частоты для каждой координаты для тех же трех датчиков. Видно, что для всех датчиков и координат увеличение погрешности наблюдалось на частотах от 3 ГГц, а для датчика с батарейным питанием (рис. 8а) также ниже 100 кГц. Рост величины ошибок измерений на частотах выше 3 ГГц обусловлен несколькими факторами:

- недостаточной изотропностью и низким уровнем симметрии относительно антенных элементов, расположенных вдоль каждой из осей X, Y иZ (для получения наиболее достоверной информации о напряженности поля монопольные антенны, встроенные в датчик, должны быть взаимно перпендикулярны с высокой точностью);

- нормированием изотропности на 10, 100 и 1000 МГц, но не выше;

- размером и формой датчика поля;

- отсутствием возможности коррекции погрешности датчика поля.

Учитывая эти недостатки, специалисты DARE!! Instruments приняли решение разработать серию датчиков RSS2010 (рис. 9), которые на сегодняшний день являются самыми точными приборами для измерения однородности поля и калибровки испытательных систем на устойчивость к РЧ-полю в диапазоне частот 9 кГц - 12 ГГц. На рис. 10 приведен график зависимости погрешности измерений от частоты для датчика поля серии RSS2010.

Потребность в приборах для проведения испытаний бортовой аппаратуры стремительно возрастает. Так как на основе активных антенных решеток стало возможно проводить испытания на восприимчивость к излучаемому
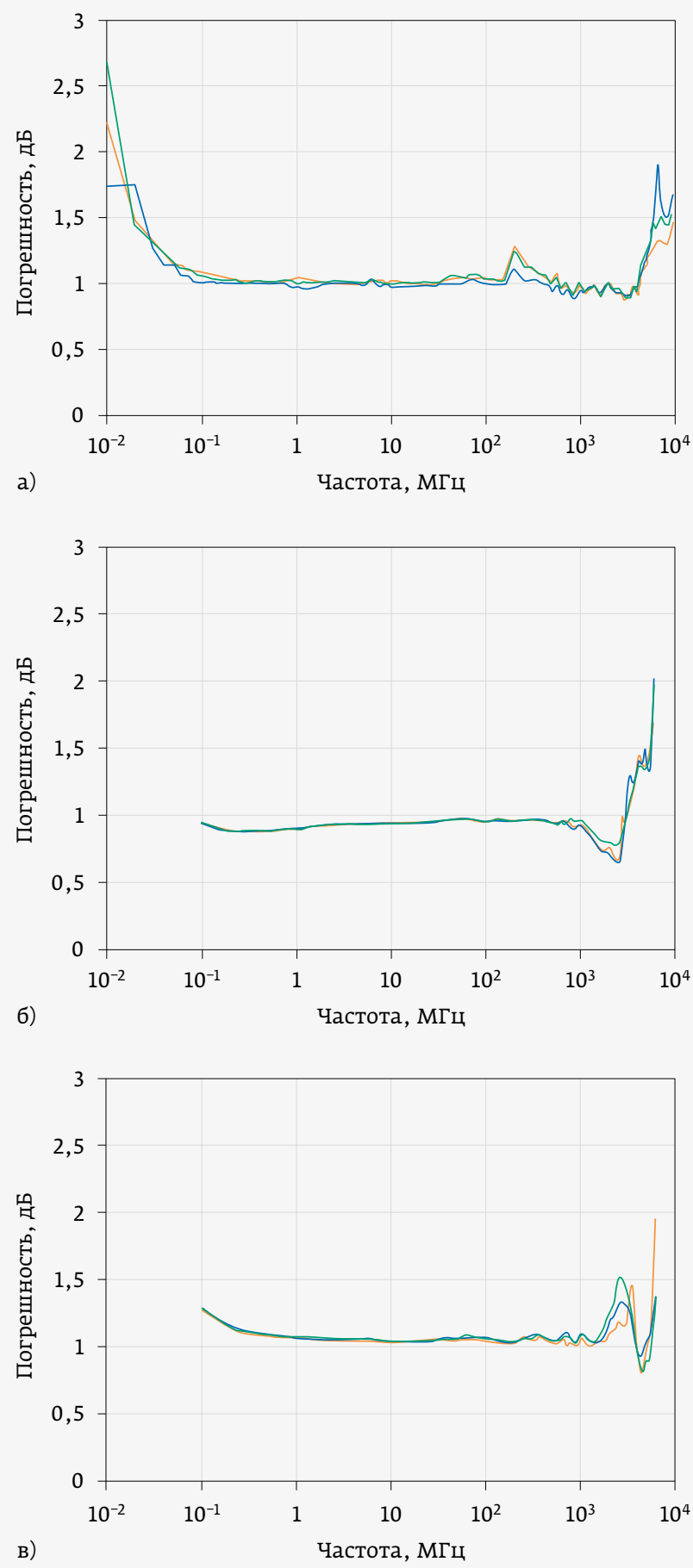

Рис. 8. Зависимости погрешности измерений от частоты для датчиков № 1 (а), № 2 (б) и № 3 (в)

ЭМ-полю, специалисты ООО «Остек-Электро» приняли решение разработать комплекс собственного производства, который дополнял бы рассмотренную измерительную систему генераторами для проведения испытаний 


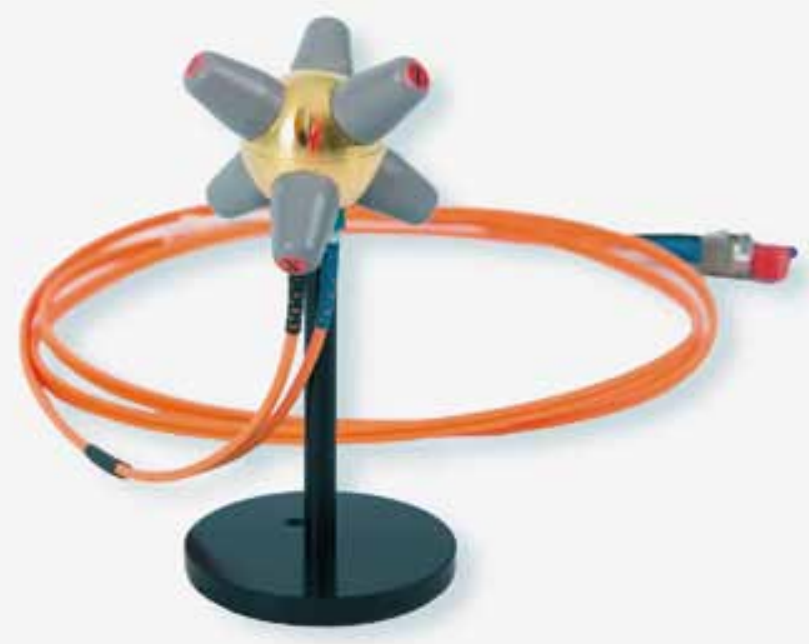

Рис. 9. Внешний вид датчика серии RSS2010

на восприимчивость к кондуктивным помехам (по стандарту MIL-STD-461G):

- НЧ синусоидальное воздействие (раздел CS101);

- в4 синусоидальное воздействие (раздел CS114);

- импульсное воздействие (раздел CS115);

- затухающее синусоидальное воздействие (раздел CS116).

Совмещение данных генераторов с генераторами поля и системами DARE!! Instruments, отвечающими требованиям разделов RS103 и CS114 стандарта MIL-STD-461G, позволяет обеспечить полное соответствие требованиям российских стандартов к проведению испытаний на ЭМС бортовой авиационной аппаратуры. Выполнение испытаний по разделам CS101, CS115 и CS116 осуществляется модульной системой собственной разработки и производства ООО "Остек-Электро" - генератором импульсным комбинированным ГИК3000 (рис. 11).

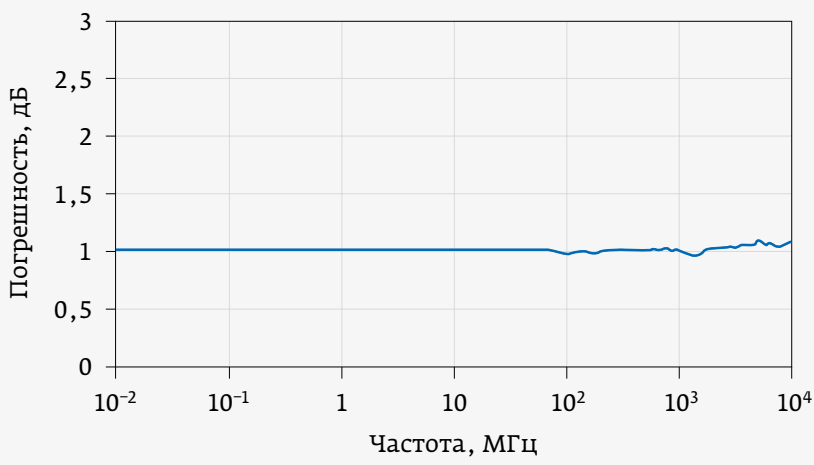

Рис. 10. Зависимости погрешности измерений от частоты для датчика поля серии RSS2010

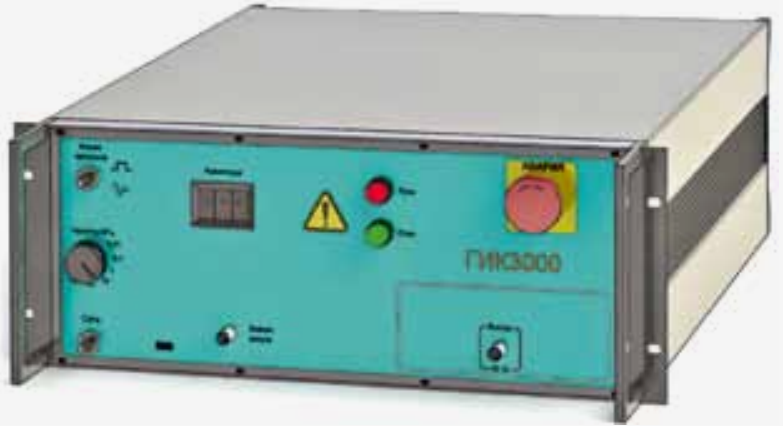

Рис. 11. Генератор ГИК3000

Помимо стандарта MIL-STD-461G, система также отвечает требованиям других отечественных и зарубежных стандартов, таких как DO-160, KT-160D (разделы 17 и 19).

Испытания по разделу CS114 возможны благодаря оборудованию, входящему в состав системы DARE!! Instruments CIT Bundle (рис. 12), представляющей собой полностью укомплектованный комплекс для выполнения испытаний на устойчивость К кондуктивным высокочастотным помехам в соответствии с требованиями стандартов IEC/ EN 61000-4-6 (ГОСТ 51317.4.6-99), MIL-STD-461G, KT-160D, ISO 11452-4. Высокочастотный генератор и высокочастотный усилитель мощности, входящие в комплекс, позволяют формировать выходные сигналы мощностью до 200 Вт и выше в диапазоне частот от 9 кГц до 400 МГц. Также возможно понижение диапазона до 4 кГц для моделирования токов, которые присутствуют в силовых кабелях аппаратуры, установленной на платформах с питанием от статических генераторов, таких как корабли и подводные лодки.

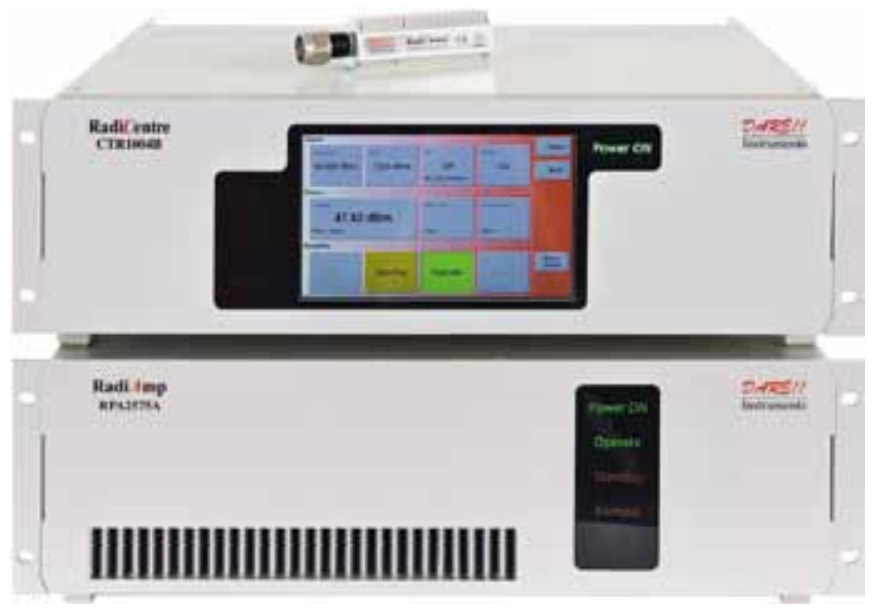

Рис. 12. Система DARE!! Instruments CIT Bundle 


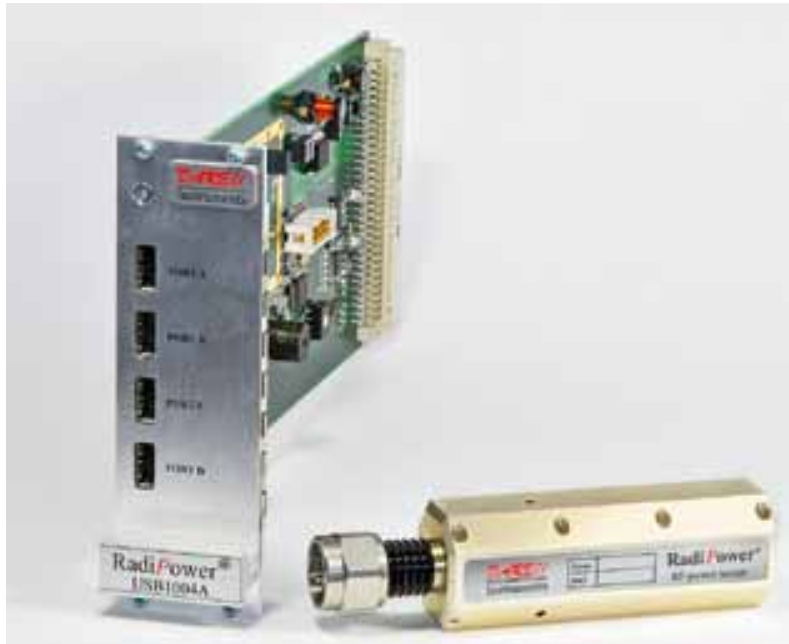

Рис. 13. Датчик мощности RPR2006CR

С помощью вспомогательного внутреннего направленного ответвителя система может измерять мощность в прямом и обратном (отраженная мощность) направлениях, используя датчики мощности RPR2006CR компании DARE!! Instruments (рис. 13), которые в диапазоне частот от 4 кГц до 6 ГГц регистрируют мощность со скоростью до 10 млн отсчетов/ с. Комплекс обеспечивает выполнение тестов в полностью автоматическом режиме в заданном диапазоне частот. Также с комплексом поставляются дополнительные опции: устройства связи/развязки, базовое шасси, программное обеспечение

Используя уникальные возможности активных антенных решеток и другой продукции DARE!! Instruments во взаимодействии с различной измерительной аппаратурой, предназначенной для испытаний на ЭМС, можно решать самые различные задачи проверки устойчивости радиоэлектронного оборудования к мощным электромагнитным воздействиям, позволяя экономить как бюджет, так и время на проведение испытаний.

Подробнее с характеристиками аппаратуры можно ознакомиться на сайте www.ostec-electro.ru.

\section{ЛИТЕРАТУРА}

1. Кондрашов Д. Зачем нужно менять устоявшиеся подходы к тестированию на ЭМс? // Вектор высоких технологий. 2016. № 6 (27). С. 66-74.

2. Кондрашов Д., Дейкстра П. Как обеспечить точность измерений напряженности поля // Вектор высоких технологий. 2018. № 3 (38). С. 54-58.

\section{НОВЫЕ КНИГИ ИЗДАТЕЛЬСТВА "ТЕХНОСФЕРА»}

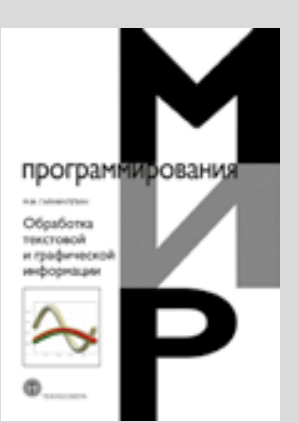

Цена 636 руб.

\section{ОБРАБОТКА ТЕКСТОВОЙ И ГРАФИЧЕСКОЙ ИНФОРМАЦИИ}

\section{М.Ф. Гарифуллин}

Рассмотрены способы обработки текстовой и графической информации. Уделено внимание вопросам сортировки текстовых данных, построения и сканирования графиков, поддержки диалогового режима работы, создания и систематизации графических файлов.

Приведены примеры текстов программ на языке FORTRAN с подробными комментариями.

Издание предназначено для научных работников и инженеров, занятых расчетами и обработкой экспериментальных данных, а также преподавателей, студентов и аспирантов технических вузов.

\section{КАК ЗАКАЗАТЬ НАШИ КНИГИ?}

$凶$ 125319, Москва, а/я 91; +7 495 234-0110; 呾+7 495 956-3346; knigi@technosphera.ru, sales@technosphera.ru 